\title{
Reply to Lewis: metaphysics versus epistemology
}

\author{
DAVID PAPINEAU \& VÍCTOR DURÀ-VILÀ
}

Peter J. Lewis (2007) argued that the Everettian interpretation of quantum mechanics implies the unpopular halfer position in the Sleeping Beauty debate. We retorted that it is perfectly coherent to be an Everettian and an ordinary thirder (Papineau and Durà-Vilà 2009). In a recent reply to our paper Lewis (2009) further clarifies the basis for his thinking. We think this brings out nicely where he goes wrong: he underestimates the importance of metaphysical considerations in determining rational credences.

Lewis's original argument hinged on the epistemic parallels between (a) an Everettian scenario where the $x$-spin of an electron in an eigenstate of $z$-spin is measured, with the result hidden from an observer and (b) a simplified Sleeping Beauty scenario where Sleeping Beauty is told that she will be woken on Monday and on Tuesday, with memory erasure in between. Lewis (2007: 60) pointed out that in both cases the agent's subjective experience has the same branching structure, with the agent becoming uncertain about her location therein. He concluded that the agent should adopt the same credences in both cases. Given that Everettians are committed in the spin case to assigning pre-measurement credences of 1/2 to 'up' and 'down', Lewis infers that the Everettian interpretation implies that Sleeping Beauty should similarly assign pre-sleeping credences of $1 / 2$ to waking on Monday and waking on Tuesday. But this would of course be very bad for the Everettian interpretation, since Sleeping Beauty's credences here should clearly be 1 .

In essence our retort was that the epistemic parallels between the two cases are trumped by their metaphysical differences. Everettians think that reality itself branches when quantum events take place. But there is no such metaphysical branching in the Sleeping Beauty case. We argued that this warrants a differential assignment of credences of $1 / 2$ in the spin case but 1 in the simplified Sleeping Beauty case.

Lewis's first response is to query the extent of the metaphysical differences:

If 'reality' is that which is described by the underlying physics, then there is no branching of reality even in the Everettian case; branching (for the contemporary Everettian) is a phenomenon that emerges at the macroscopic level via decoherence. That is, in the Everettian case as in the (simplified) Sleeping Beauty case, there is a single world in which the agent is uncertain of her location. (2009: ??)

We are happy to grant that in one sense there is no branching for Everettians. There is no branching at the level of the underlying physics. But in another sense the Everettian interpretation clearly does postulate branching. Everettians hold that after the measurement decoherence generates a part of reality that behaves like a quasiclassical world and contains a post-fission agent who sees spin-up, and another part of reality that behaves analogously and contains a post-fission agent who sees spin-down. 
From this perspective it is misleading to talk of a 'single world' in both the Everettian and simplified Sleeping Beauty cases. Where the single world containing Sleeping Beauty is metaphysically familiar, the Everettian world is a multiverse containing many separate branches of reality resulting from decoherence.

Still, we needn't fight about the terminology of 'branching'. For Lewis quickly moves onto another tack. He says that he is happy to allow that there are genuine metaphysical differences between the two cases. But he then argues that these metaphysical differences can't really matter, given that the two cases are carefully constructed to be epistemically parallel.

This is where we think Lewis goes wrong: he is here assuming that the rational assignments of credences can be settled independently of metaphysics. But we see no reason for this assumption. After all, on the conventional interpretation of quantum mechanics, nobody thinks that the assignment of credences to quantum results is independent of metaphysical matters. On the contrary, it is universally agreed that the rational strategy is to match your credences to the objective quantum probabilities, in line with David Lewis's (1986: 87) Principal Principle.

Everettians will agree that subjects should match their degrees of belief to the objective quantum probabilities. The issue is then where such probabilities are to be found. Everettians say that they arise specifically when decoherence yields quasiclassical branches of reality, and moreover that they are equal to the squared moduli of the amplitudes of those branches.

Against this background, it is irrelevant that there are certain experiential parallels between simplified Sleeping Beauty and Everettian spin measurements. Everettians will simply respond that the Sleeping Beauty scenario does not involve the kind of metaphysical structure that gives rise to non-unitary quantum probabilities, for lack of any decoherent branching.

Since there is no decoherent branching in the Sleeping Beauty case, the objective probabilities of waking on Monday and Tuesday are both 1 . In the spinmeasurement case, by contrast, there is branching, with the squared moduli of the amplitudes of each branch equal to $1 / 2$. The rational degrees of belief follow suit, in line with the Principal Principle. The point is that rational subjective probabilities here track objective ones, and the objective probabilities depend on the metaphysical structure. Far from being irrelevant, the metaphysical structure is crucial to the determination of rational degrees of belief. ${ }^{1,2}$

\footnotetext{
${ }^{1}$ In our earlier paper (2009: §7) we offered Lewis the support of Lev Vaidman's approach to Everettian probability $(1998,2002)$. Lewis declined this offer, querying our assumption that Vaidman really aims to derive pre-fission uncertainty from post-fission subjects' ignorance about location. We still find it hard to read Vaidman any other way. (Consider for instance his claim that the pre-fission agent 'can associate probability for different outcomes according to the ignorance probability of each of his descendants' 1998: 254.)

${ }^{2}$ We are grateful to Peter J. Lewis, Darren Bradley and Paul Tappenden for discussion of these issues.
} 
King's College London Strand, London WC2R 2LS, UK david.papineau@kcl.ac.uk

victor.dura_vila@kcl.ac.uk

\section{References}

Lewis, D. 1986/1980. A subjectivist's guide to objective chance. In his Philosophical Papers: Volume II, 83-132. New York: Oxford University Press.

Lewis, P. J. 2007. Quantum Sleeping Beauty. Analysis 67: 59-65.

Lewis, P. J. 2009. Reply to Papineau and Durà-Vilà. Analysis 69: ??-??.

Papineau, D. and V. Durà-Vilà. 2009. A thirder and an Everettian: a reply to Lewis's 'Quantum Sleeping Beauty'. Analysis 69: ??-??.

Vaidman, L. 1998. On schizophrenic experiences of the neutron or why we should believe in the many-worlds interpretation of quantum theory. International Studies in the Philosophy of Science 12: 245-61.

Vaidman, L. 2002. Many-worlds interpretation of quantum mechanics. In The Stanford Encyclopedia of Philosophy, ed. E. N. Zalta.

http://plato.stanford.edu/entries/qm-manyworlds/. Accessed 17 December 2007. 